\title{
BMR
}

\section{Association between monoamine oxidase B A644G polymorphism and Parkinson's disease risk: a meta-analysis in the Chinese population}

\author{
J.J. Liu ${ }^{1}$, W. Wang ${ }^{1}$, M. Meng ${ }^{1}$, C.S. Liang ${ }^{2}$ and J.W. Zhang ${ }^{1}$ \\ ${ }^{1}$ Department of Neurology, \\ The First Affiliated Hospital of Qiqihar Medical University, \\ Qiqihar, Heilongjiang, China \\ ${ }^{2}$ Department of Clinical Medicine, \\ Qiqihar Medical University, Qiqihar, Heilongjiang, China \\ Corresponding author: J.W. Zhang \\ E-mail: wjzhang668@126.com
}

Genet. Mol. Res. 15 (2): gmr.15028349

Received December 28, 2015

Accepted February 11, 2016

Published July 15, 2016

DOI http://dx.doi.org/10.4238/gmr.15028349

\begin{abstract}
Although various individual studies have evaluated the correlation between monoamine oxidase B (MAOB), polymorphism, and Parkinson's disease (PD), the results remain inconclusive. Therefore, we performed a meta-analysis in the Chinese population to provide comprehensive data on the association between the MAOB polymorphism and PD. Eligible studies were identified via databases such as PubMed, Springer Link, Ovid, Chinese Wanfang Data Knowledge Service Platform, Chinese National Knowledge Infrastructure, and Chinese Biology Medicine, throughout November 2015. Pooled odds ratios (ORs) and 95\% confidence intervals (CIs) were used to assess the strengths of these associations. Eight studies documenting a total of 1385 cases of PD and 1426 controls were
\end{abstract}


included in this meta-analysis. Overall, no significant association was found between the MAOB A644G polymorphism and PD risk in the Chinese population. However, in subgroup analyses, where results were stratified by geographical areas and source of controls, increased risk for PD in Northern China was observed (allele A vs G: OR $=1.33$, $95 \% \mathrm{CI}=1.11-1.58 ; \mathrm{AA} v s \mathrm{GG}: \mathrm{OR}=1.46,95 \% \mathrm{CI}=1.09-1.97 ; \mathrm{AA}$ + AG vs GG: $\mathrm{OR}=1.42,95 \% \mathrm{CI}=1.06-1.90)$. Similarly, populationbased studies also showed significant association between the MAOB A644G polymorphism and PD risk among different populations (allele A $v s$ G: $\mathrm{OR}=1.29,95 \% \mathrm{CI}=1.11-1.51$; AA $v s \mathrm{GG}$ : $\mathrm{OR}=1.41$, $95 \% \mathrm{CI}=1.09-1.82$; $\mathrm{AA}+\mathrm{AG} v s \mathrm{GG}: \mathrm{OR}=1.34,95 \% \mathrm{CI}=1.04-$ 1.71). In conclusion, this meta-analysis provided evidence that the MAOB A644G polymorphism may contribute to PD development in Northern China. Further studies conducted in other ethnic groups are required for definite conclusions.

Key words: Meta-analysis; Monoamine oxidase B; Polymorphism; Parkinson's disease

\section{INTRODUCTION}

Parkinson's disease (PD), a common neurodegenerative disorder characterized by bradykinesia, muscle rigidity, postural instability, and resting tremor, is the second most prevalent neurodegenerative disease after Alzheimer's disease, and affects more than $1 \%$ of the elderly population (Löhle and Reichmann, 2011; Lieu et al., 2013; Chen et al., 2015). A previous study estimated that China has 2 million people with $\mathrm{PD}$, which is approximately $48 \%$ of all individuals with PD in the world's 15 most populous countries (Dorsey et al., 2007); this number is expected to increase to 5 million by the year 2030 (Chen et al., 2015). Despite recent therapeutic advances, there is currently no preventive or curative therapy for PD. The exact etiology of PD remains poorly understood; however, it is recognized that genetic factors play an important role in its pathogenesis.

Many common low-penetrant genes have been identified as potential PD susceptibility genes. Of these genes, an important one is monoamine oxidase B (MAOB), which is potentially relevant to PD due to its role in catabolism of dopamine and generation of reactive oxidative free radicals (Riederer et al., 1989; Fahn and Cohen, 1992). The MAOB gene is located on the $\mathrm{X}$ chromosome. Its single-stranded conformational polymorphism occurs in intron 13, and leads to a transitional conversion from adenine (A) to guanine $(\mathrm{G})$ $36 \mathrm{bp}$ upstream from the 5'-end of exon 14 (A644G, rs1799836). An association between the MAOB polymorphism and PD in the Caucasian population was first reported by Kurth et al. (1993). Many studies have thereafter attempted to clarify this relationship; however, no definite consensus has been reached. Differences in study results may be due to ethnic and clinical heterogeneity of the patients studied, as well as the limited number of patients included in each study. Meta-analysis combines the available evidence from a number of studies, which may provide a robust result. Therefore, to address the associations between the MAOB polymorphism and PD risk, we performed a meta-analysis of all eligible studies performed in the Chinese population. 


\section{MATERIAL AND METHODS}

\section{Search strategy and selection criteria}

We searched for publications that investigated the association between the MAOB polymorphism and PD using databases such as PubMed, Springer Link, Ovid, Chinese Wanfang Data Knowledge Service Platform, Chinese National Knowledge Infrastructure, and Chinese Biology Medicine. The search terms used were as follows: 1) monoamine oxidase B, MAOB; 2) Parkinson disease; and 3) Chinese, China, Taiwan. Search terms were also combined, to generate more results. Additional studies were identified via manual screening of the reference lists from the original studies or review articles. No publication date or language restrictions were applied during article selection.

Our inclusion criteria were as follows: 1) the case-control or cohort study describes the association between the MAOB A644G polymorphism and PD;2) the study provides the distribution of the MAOB A644G polymorphism in patients and controls; 3 ) the study consists of Chinese participants only. Studies were excluded if they 1) were duplicate publications; 2) were meta-analyses, letters, meeting abstracts, reviews, or editorial articles, 3) had incomplete data, 4) or lacked controls.

\section{Data extraction}

Data from all included publications were extracted by 2 independent investigators, and a consensus was reached following discussions. Titles and abstracts of all potentially relevant articles were screened to determine their relevance. Full articles were examined if the title and abstract were ambiguous. Data were recorded as follows: first author's surname, year of publication, geographic areas, source of controls, sample size, and number of subjects with MAOB A644G genotypes.

\section{Statistical analysis}

Statistical analysis was conducted using the Stata 10.0 software (StataCorp., College Station, TX, USA), and $\alpha=0.05$ was applied as the significance level. The strength of association was estimated by means of odds ratio (OR) and its $95 \%$ confidence interval (CI). The statistical significance of pooled ORs was examined by the Z-test. Genetic heterogeneity was evaluated by $\mathrm{Q}$-tests with $\mathrm{P}<0.10$. The Hardy-Weinberg equilibrium of control subjects was calculated using the goodness-of-fit test, and a deviation was defined when $\mathrm{P}<0.05$. We used the fixed-effect model and the random-effect model based on the Mantel-Haenszel and the DerSimonian and Laird method, respectively, to evaluate the sensitivity of our analysis. To explore potential sources of heterogeneity, stratified analyses according to geographic areas and source of controls were also performed.

\section{RESULTS}

\section{Description of included studies}

Figure 1 illustrates the literature search process in the form of a flow chart. We 
identified 128 articles that investigated the association between the MAOB polymorphism and risk of PD in various databases. After screening the titles and abstracts, 117 articles were excluded according to the exclusion criteria described. Of the 11 potentially relevant articles identified for full-study retrieval (Hwang et al., 1997; Shao et al., 2001; Wu et al., 2001; Jiang et al., 2004; Gu et al., 2010; Wang and Zhang, 2010; Li, 2011; Zeng et al., 2012; Zhang et al., 2013; Hao et al., 2015; Song et al., 2015), 3 were excluded owing to being duplicate studies or lacking controls (Zeng et al., 2012; Hao et al., 2015; Song et al., 2015). Finally, 8 studies (Hwang et al., 1997; Shao et al., 2001; Wu et al., 2001; Jiang et al., 2004; Gu et al., 2010; Wang and Zhang, 2010; Li, 2011; Zhang et al., 2013) met the inclusion criteria. The publication years of the studies ranged from 1997 to 2013. In total, 1385 cases of PD and 1426 controls were included in this meta-analysis. The characteristics of these studies are summarized in Table 1.

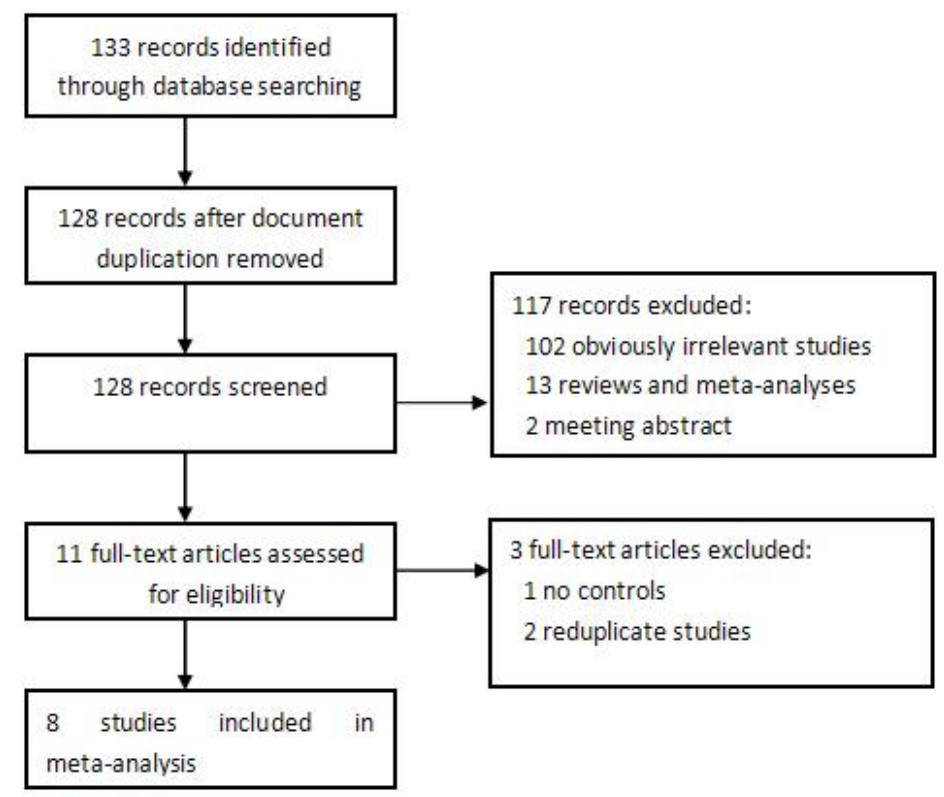

Figure 1. Flow diagram of the literature search.

Table 1. Characteristics of studies included in the meta-analysis.

\begin{tabular}{|c|c|c|c|c|c|c|c|c|c|c|c|c|}
\hline \multirow[t]{2}{*}{ References (first author) } & \multirow[t]{2}{*}{ Source of controls } & \multirow[t]{2}{*}{ Geographic areas } & \multirow[t]{2}{*}{ Cases $(\mathrm{N})$} & \multirow[t]{2}{*}{ Controls $(\mathrm{N})$} & \multicolumn{3}{|c|}{ Cases } & \multicolumn{3}{|c|}{ Controls } & \multicolumn{2}{|c|}{ HWE } \\
\hline & & & & & AA & $\mathrm{AG}$ & GG & AA & AG & GG & $\chi^{2}$ & $\mathrm{P}$ value \\
\hline Hwang, 1997 & PB & Taiwan & 65 & 108 & 49 & 11 & 5 & 76 & 19 & 13 & 23.52 & 0.00 \\
\hline Shao, 2001 & PB & Guangdong & 126 & 136 & 65 & 25 & 36 & 62 & 34 & 40 & 32.20 & 0.00 \\
\hline $\mathrm{Wu}, 2001$ & $\mathrm{~PB}+\mathrm{HB}$ & Taiwan & 220 & 191 & 169 & 14 & 37 & 158 & 16 & 17 & 76.24 & 0.00 \\
\hline Jiang, 2004 & PB & Beijing & 266 & 154 & 207 & 31 & 28 & 114 & 13 & 27 & 87.09 & 0.00 \\
\hline $\mathrm{Gu}, 2010$ & $\mathrm{~PB}$ & Beijing & 176 & 354 & 153 & \multicolumn{2}{|c|}{23} & 323 & \multicolumn{2}{|c|}{31} & & \\
\hline Wang, 2010 & PB & Tianjin & 125 & 66 & 88 & 23 & 14 & 34 & 19 & 13 & 8.52 & 0.00 \\
\hline $\mathrm{Li}, 2011$ & PB & Hebei & 166 & 170 & 111 & 24 & 31 & 103 & 30 & 37 & 58.06 & 0.00 \\
\hline Zhang, 2013 & PB & Xinjiang & 241 & 247 & 177 & 34 & 30 & 179 & 32 & 36 & 91.99 & 0.00 \\
\hline
\end{tabular}

$\mathrm{PB}=$ population-based; $\mathrm{HB}=$ hospital-based. 


\section{Meta-analysis}

Table 2 lists the primary results of our meta-analysis. We did not find elevated PD risk in any of the evaluated models. However, when we stratified our analysis based on geographical areas, increased PD risk was found in individuals from Northern China (A vs $\mathrm{G}: \mathrm{OR}=1.33,95 \% \mathrm{CI}=1.11-1.58 ; \mathrm{AA} v s \mathrm{GG}: \mathrm{OR}=1.46,95 \% \mathrm{CI}=1.09-1.97 ; \mathrm{AA}+\mathrm{AG} v s$ GG: $\mathrm{OR}=1.42,95 \% \mathrm{CI}=1.06-1.90)$; however, this increase was not observed in those from Southern China. In the subgroup analysis based on source of controls, significant association was found in population-based studies (A vs $\mathrm{G}: \mathrm{OR}=1.29,95 \% \mathrm{CI}=1.11-1.51$; $\mathrm{AA}$ vs GG: OR $=1.41,95 \% \mathrm{CI}=1.09-1.82 ; \mathrm{AA}+\mathrm{AG}$ vs $\mathrm{GG}: \mathrm{OR}=1.34,95 \% \mathrm{CI}=1.04-1.71)$.

\begin{tabular}{|c|c|c|c|c|c|}
\hline Analysis model & & $\mathrm{OR}_{\mathrm{r}}(95 \% \mathrm{CI})$ & $\mathrm{OR}_{\mathrm{f}}(95 \% \mathrm{CI})$ & $\mathrm{P}_{\mathrm{h}}$ & \\
\hline \multirow[t]{4}{*}{$\mathrm{A} v s \mathrm{G}$} & Total analysis & 7 & $1.19(0.92-1.53)$ & $1.16(1.00-1.33)$ & 0.005 \\
\hline & Population-based & 6 & $1.30(1.11-1.52)$ & $1.29(1.11-1.51)$ & 0.396 \\
\hline & Southern China & 3 & $0.96(0.59-1.58)$ & $0.92(0.73-1.16)$ & 0.016 \\
\hline & Northern China & 4 & $1.35(1.08-1.69)$ & $1.33(1.11-1.58)$ & 0.201 \\
\hline \multirow[t]{4}{*}{$\mathrm{AA} v s \mathrm{GG}$} & Total analysis & 7 & $1.24(0.87-1.76)$ & $1.19(0.94-1.50)$ & 0.047 \\
\hline & Population-based & 6 & $1.41(1.09-1.82)$ & $1.41(1.09-1.82)$ & 0.685 \\
\hline & Southern China & 3 & $0.92(0.45-1.85)$ & $0.86(0.59-1.25)$ & 0.057 \\
\hline & Northern China & 4 & $1.47(1.09-1.98)$ & $1.46(1.09-1.97)$ & 0.476 \\
\hline \multirow{4}{*}{$\mathrm{AA} v s \mathrm{AG}$} & Total analysis & 7 & $1.19(0.93-1.53)$ & $1.19(0.93-1.52)$ & 0.483 \\
\hline & Population-based & 6 & $1.19(0.90-1.57)$ & $1.18(0.91-1.54)$ & 0.359 \\
\hline & Southern China & 3 & $1.28(0.84-1.93)$ & $1.28(0.84-1.93)$ & 0.887 \\
\hline & Northern China & 4 & $1.17(0.76-1.76)$ & $1.14(0.84-1.55)$ & 0.166 \\
\hline \multirow{4}{*}{$\mathrm{AA} v s(\mathrm{AG}+\mathrm{GG})$} & Total analysis & 8 & $1.12(0.87-1.44)$ & $1.11(0.93-1.33)$ & 0.062 \\
\hline & Population-based & 7 & $1.20(0.95-1.53)$ & $1.20(0.99-1.44)$ & 0.159 \\
\hline & Southern China & 3 & $1.02(0.66-1.55)$ & $1.00(0.73-1.36)$ & 0.163 \\
\hline & Northern China & 5 & $1.18(0.85-1.64)$ & $1.17(0.95-1.45)$ & 0.058 \\
\hline \multirow[t]{4}{*}{$(\mathrm{AA}+\mathrm{AG}) v s \mathrm{GG}$} & Total analysis & 7 & $1.18(0.84-1.66)$ & $1.14(0.91-1.44)$ & 0.055 \\
\hline & Population-based & 6 & $1.34(1.04-1.72)$ & $1.34(1.04-1.71)$ & 0.678 \\
\hline & Southern China & 3 & $0.86(0.45-1.66)$ & $0.82(0.57-1.18)$ & 0.073 \\
\hline & Northern China & 4 & $1.42(1.06-1.91)$ & $1.42(1.06-1.90)$ & 0.575 \\
\hline
\end{tabular}

$\mathrm{OR}_{\mathrm{r}}=$ odds ratio for random-effect model; $\mathrm{OR}_{\mathrm{f}}=$ odds ratio for fixed-effect model; $\mathrm{P}_{\mathrm{h}} \mathrm{P}$ value for the heterogeneity test; Northern China included Beijing, Tianjin, Hebei, Xinjiang; Southern China included Guangdong, Taiwan.

\section{Sensitive analysis}

In order to evaluate the sensitivity of the analysis, we used the fixed-effect and random-effect model to determine the stability of our meta-analysis. None of the results were materially altered in either the total or the subgroup analysis (Table 2), suggesting that the data included in this study are relatively stable and reliable.

\section{DISCUSSION}

Several studies have offered evidence that individual susceptibility to PD is partially determined by genetic predisposition. The relationship between the MAOB A644G polymorphism and PD risk attracted the attention of both doctors and researchers. To date, 3 meta-analyses regarding the MAOB A644G polymorphism and PD risk have been published (Liu et al., 2014; Sun et al., 2014; Zhang et al., 2015). Of these, 1 meta-analysis reported that 
there was a significant association between the MAOB A644G polymorphism and PD risk in both Asians and Caucasians (Liu et al., 2014). However, the other 2 studies suggested that there was no significant association between the MAOB polymorphism and PD risk among Caucasians (Sun et al., 2014; Zhang et al., 2015). It is possible that regional and racial differences have contributed to the conflicting results. Therefore, we conducted this meta-analysis to provide a more precise estimation of the association between the MAOB A644G polymorphism and PD susceptibility in only the Chinese population in order to reduce the effect of regional and racial differences.

In the overall analysis, no significant association was found between the MAOB A644G polymorphism and PD in the Chinese population. To examine whether environmental risk factors can modulate PD risk, subgroup analyses stratified by geographical areas and sources of controls were performed. We found that the MAOB A644G polymorphism significantly increased the risk of PD in Northern China, and similar results were observed in population-based studies; in contrast, this increase was not observed in Southern China. This result suggested that differences in genetic backgrounds as well as in the environment may influence the association between the MAOB A644G polymorphism and PD risk.

As compared to previous meta-analyses (Liu et al., 2014; Sun et al., 2014; Zhang et al., 2015), the current study included more researches conducted in the Chinese population. Furthermore, our study has higher statistical power than that of previous metaanalyses conducted in other ethnic groups. The effects of gene-environment interactions with respect to PD risk were also determined by subgroup analyses. Sensitivity analyses confirmed the reliability and stability of the meta-analysis. Therefore, our results indicated that the MAOB A644G polymorphism is associated with PD in individuals from Northern China.

Several limitations were present in our meta-analysis. First, this ethnic-specific meta-analysis only included data from Chinese patients, and thus, our results are only applicable to this ethnic group. Second, because this meta-analysis was primarily based on unadjusted effect estimates and CIs, we did not control for confounding factors. Third, results from the Hardy-Weinberg equilibrium test for genotype distribution in the control groups suggested that significant differences in genetic background existed among the subjects. Finally, due to the limitations in funnel plotting, which requires a range of studies, we did not evaluate publication bias in this meta-analysis.

In conclusion, our meta-analysis results support the hypothesis that the MAOB A644G polymorphism may contribute to individual susceptibility to PD in Northern China. Further studies are required to determine whether the MAOB A644G polymorphism confers PD risk in other ethnic groups.

\section{Conflicts of interest}

The authors declare no conflict of interest.

\section{ACKNOWLEDGMENTS}

Research supported by the Heilongjiang Association of Higher Education, China, 2014 Higher Education Scientific Research topics (\#14G160). 


\section{REFERENCES}

Chen H, Ding D, Wang J, Zhao Q, et al. (2015). Parkinson's disease research in a prospective cohort in China. Parkinsonism Relat. Disord. 21: 1200-1204. http://dx.doi.org/10.1016/j.parkreldis.2015.08.020

Dorsey ER, Constantinescu R, Thompson JP, Biglan KM, et al. (2007). Projected number of people with Parkinson disease in the most populous nations, 2005 through 2030. Neurology 68: 384-386. http://dx.doi.org/10.1212/01. wnl.0000247740.47667.03

Fahn S and Cohen G (1992). The oxidant stress hypothesis in Parkinson's disease: evidence supporting it. Ann. Neurol. 32: 804-812. http://dx.doi.org/10.1002/ana.410320616

Gu Z, Feng X, Dong X and Chan P (2010). Smoking, genes encoding dopamine pathway and risk for Parkinson's disease. Neurosci. Lett. 482: 31-34. http://dx.doi.org/10.1016/j.neulet.2010.06.085

Hao H, Shao M, An J, Chen C, et al. (2015). [Polymorphisms of catechol-O-methyltransferase and monoamine oxidase B genes among Chinese patients with Parkinson's disease]. Zhonghua Yi Xue Yi Chuan Xue Za Zhi 32: 1-5.

Hwang WJ, Lai ML, Tsai TT and Lai MD (1997). Genetic polymorphism of monoamine oxidase B and susceptibility of Parkinson's disease. Zhonghua Yi Xue Za Zhi (Taipei) 60: 137-141.

Jiang XH, Xu QY, Yang H and Chen B (2004). Relationship between polymorphism of monoamine oxidase type B gene and Parkinson's disease in Chinese. Chin. J. Neurol. 37: 239-242.

Kurth JH, Kurth MC, Poduslo SE and Schwankhaus JD (1993). Association of a monoamine oxidase B allele with Parkinson's disease. Ann. Neurol. 33: 368-372. http://dx.doi.org/10.1002/ana.410330406

Li W (2011). Relationship between polymorphism of monoamine oxidase B gene intron $13 \mathrm{G} / \mathrm{A}$ and Parkinson disease. J. Clin. Neurol. 24: 261-263.

Lieu CA, Chinta SJ, Rane A and Andersen JK (2013). Age-related behavioral phenotype of an astrocytic monoamine oxidase-B transgenic mouse model of Parkinson's disease. PLoS One 8: e54200. http://dx.doi.org/10.1371/journal. pone. 0054200

Liu Y, Wang Z and Zhang B (2014). The relationship between monoamine oxidase B (MAOB) A644G polymorphism and Parkinson disease risk: a meta-analysis. Ann. Saudi Med. 34: 12-17.

Löhle M and Reichmann H (2011). Controversies in neurology: why monoamine oxidase B inhibitors could be a good choice for the initial treatment of Parkinson's disease. BMC Neurol. 11: 112. http://dx.doi.org/10.1186/1471-2377$11-112$

Riederer P, Konradi C, Hebenstreit G and Youdim MB (1989). Neurochemical perspectives to the function of monoamine oxidase. Acta Neurol. Scand. Suppl. 126 (Suppl.): 41-45. http://dx.doi.org/10.1111/j.1600-0404.1989.tb01781.x

Shao M, Liu Z, Tao E and Chen B (2001). [Polymorphism of MAO-B gene and NAD(P)H: quinone oxidoreductase gene in Parkinson's disease]. Zhonghua Yi Xue Yi Chuan Xue Za Zhi 18: 122-124.

Song QX, Li YY and Zhang XY (2015). Relationship between Xinjiang region Parkinson's disease and the interaction between polymorphisms of catechol-O-methyltransferase, monoamine oxidase B, dopamine b-hydroxylase and environmental factors. Chin. J. Clin. Neurosci. 23: 497-508.

Sun YX, Wang XH, Xu AH and Zhao JH (2014). Functional polymorphisms of the MAO gene with Parkinson disease susceptibility: a meta-analysis. J. Neurol. Sci. 345: 97-105. http://dx.doi.org/10.1016/j.jns.2014.07.016

Wang XF and Zhang BS (2010). Study of association between polymorphisms of monoamine oxidase band early-onset Parkinson's disease. Chin. J. Neurol. 43: 388-393.

Wu RM, Cheng CW, Chen KH, Lu SL, et al. (2001). The COMT L allele modifies the association between MAOB polymorphism and PD in Taiwanese. Neurology 56: 375-382. http://dx.doi.org/10.1212/WNL.56.3.375

Zeng WJ, Zhang XY, Li YY, Liu Y, et al. (2012). Relationship between polymorphism of monoamine oxidase B gene and Parkinson disease in Xinjiang Uygurs. Clin. Focus 27: 764-766.

Zhang XY, Li YY, Zeng WJ, Han XH, et al. (2013). Relationship between polymorphism of monoamine oxidase B gene and Parkinson's disease in Xinjiang, China of Uyghurs and Hans. Chin. J. Clin. Neurosci. 21: 394-400.

Zhang Y, Piao X, Wu J, Li Y, et al. (2015). A meta-analysis on relationship of MAOB intron 13 polymorphisms, interactions with smoking/COMT H158L polymorphisms with the risk of PD. Int. J. Neurosci. 20: 1-8. 